\title{
Concerning the Mechanism of Recovery in Stroke Hemiphegia
}

\author{
C. Miller Fisher
}

\begin{abstract}
Two patients with pure motor hemiplegia were regaining strength in the affected limbs when a pure motor stroke developed on the opposite side. At the same time as the new hemiplegia appeared, the recovering side became re-paralyzed, suggesting that activity in the contralateral corticospinal tract had participated in the recovery process. Pathological studies were confirmatory. The literature pertaining to contralateral motor compensation is reviewed.
\end{abstract}

RÉSUMÉ: À propos du mécanisme de récupération dans l'hémiplégie. Deux patients avec une hémiplégie motrice pure récupéraient des forces dans les membres atteints quand un ictus moteur pur s'est développé du côté opposé. Au moment où la nouvelle hémiplégie est apparue, le côté qui récupérait est devenu de nouveau paralysé, suggérant que l'activité dans le faisceau pyramidal contralatéral avait participé au processus de récupération. Les études anatomopathologiques ont confirmé cette observation. Nous revoyons la littérature concernant la compensation motrice contralatérale.

Can. J. Neurol. Sci. 1992; 19: 57-63

Recovery of motor function after a hemiplegic stroke is highly variable and the determining factors are largely unknown. When a satisfactory recovery does occur the underlying responsible mechanism also remains an unsettled question. It is commonly postulated that the contralateral corticospinal system assumes the added function just as it seems to do in the case of recovery of those parts with bilateral cerebral innervation - eye movements, face, tongue, jaw, neck, axial musculature and respiration. A number of other mechanisms of recovery exist, some of which may well be applicable while some are still for the most part theoretical: 1) the recovery from reversible local ischemic damage to neurons; 2) the resorption of edema; 3 ) the assumption of function by neighboring cortex; 4) the emergence of activity mediated by subcortical neurons; 5) the induction of synaptic sprouting; 6) the recruitment of previously ineffective or dormant synapses; 7) subcellular plasticity; and 8) regeneration of neurons. Recovery from hemiplegia is but part of the broader theme - the reorganization of neural activity after an insult, i.e., the adaptive response, the plasticity of the nervous system.

The present paper is concerned with providing additional evidence that the contralateral healthy corticospinal system contributes importantly to recovery. It is a common clinico-pathological finding that after a hemiplegic stroke recovery of motor function may have been quite good, yet on pathologic examination the involved corticospinal system shows severe degeneration and atrophy, forcing the conclusion that recovery must have been due to activity in some other system, the main candidate for this being the opposite corticospinal system. However, uncertainty remained and in addition residual function in the involved corticospinal system could not be excluded. What has been missing is a case in which a second hemiplegia occurs on the opposite side, involving the corticospinal system that now presumably is sustaining the motor activity of both sides of the body. If recovery occurred via the contralateral motor system destruction of the second corticospinal system should not only cause paralysis of the corresponding limbs but should also result in re-paralysis of the recovered limbs of the first hemiplegia. This is what happened in the two patients to be described here. Both had suffered two hemiplegias, the first involving the limbs of the left side which recovered some function whereupon a second hemiplegia involving the right side resulted in bilateral paralysis.

The literature contains only two reports in which comparable evidence is presented. Maspes and Pagni ${ }^{2}$ performed staged surgical pedunculotomies bilaterally in two young patients with severe choreoathetosis. After the first operation the opposite limbs were immediately paralyzed but made some recovery only to become paralyzed again as a result of the pedunculotomy on the other side, the second operation producing, in effect, a tetraplegia. The authors suggested that in addition to recovery due to incomplete incision of the peduncular fibers some recovery may have been partly produced by fibers descending from the opposite motor cortex. There was also the reservation that the neural organization in cerebral palsy is probably abnormal.

From the Neurology Service of the Massachusetts General Hospital, Fruit Street, Boston

Received April 2, 1991. Accepted in final form September 16, 1991

Reprint requests to: Dr. C. Miller Fisher, Neurology Service of the Massachusetts General Hospital, Fruit Street, Boston, Massachusetts, U.S.A. 02114 
The second report concerned lesions of the spinal cord and was that of Nathan and Smith. ${ }^{3}$ In eight patients they showed that when paralysis of a leg occurred as a result of unilateral spinothalamic cordotomy at the thoracic level, motor recovery was the rule. This recovery was lost and a paraplegia resulted when a second spinothalamic cordotomy performed on the opposite side extended posteriorly to involve the second corticospinal system. Analysis of the pathological studies of the spinal cords showed it was involvement of the lateral corticospinal tracts that was responsible.

The present report is timely in the light of recent studies of regional cerebral blood flow (CBF) in recovered monoplegics. ${ }^{4}$ Using positron emission tomography (PET) scanning, the effect of finger movements on CBF was studied comparing the normal hand with the recovered hand. Movements of the fingers on the normal hand were associated with increased blood flow mainly in the contralateral cerebral hemisphere. Movements of the fingers of the recovered hand were associated with increased flow in both hemispheres, that is activity in the healthy hemisphere accompanied movements of the recovered limb.

Experimental operations in animals have given results that accord only partly with clinical experience as far as the brain is concerned whereas the findings in the case of the spinal cord are comparable.

\section{Report of Cases}

Patient $1^{5}$ A mildly diabetic hypertensive woman, aged 75 , rather abruptly noted weakness of her left hand and leg and fell to the floor. Ten hours later she managed to kick the telephone receiver off the hook and notify the operator. On admission to the hospital 12 hours from the onset there was moderate weakness of the left side of the face and of the left arm and leg. In the following 48 hours progression occurred and examination showed a flaccid palsy of the left upper extremity. The left leg could be lifted in the air and the toes flexed and extended slowly without power. Eye movements were full without nystagmus. There was no dysarthria or visual field defect. Sensation was intact for pinprick, vibration, touch, joint position, stereognosis and number-writing. The tendon reflexes were brisk bilaterally. A Babinski sign was present on the left. Spasmodic laughing and crying were observed. The blood pressure was $170 / 90$.

Three days after admission the left thumb moved slightly and the passively abducted left arm could be slightly adducted. After 5 days the left index and middle fingers moved weakly and pronation of the forearm appeared. The patient walked $20 \mathrm{~m}$ with assistance lifting the left leg well. After 12 days the left hand grip was improving and abduction at the shoulder was possible. After 19 days the left grip was stronger, dorsiflexion at the ankle was returning and the patient walked $40 \mathrm{~m}$, with one assistant. Weakness of the left side of the face had decreased and spasms of laughing and crying were fewer.

On the 20th hospital day at 1600 hours the patient became almost mute for about five minutes. This recurred at 1730 hours. Examination at 1800 hours showed no change from her previous state. On day 22 she had three five-minute attacks of speechlessness and complete paralysis of the right arm and leg. Each spell started with slight numbness of the right fingers. Examination during the attack showed drooling, retention of alertness and comprehension, and bilateral Babinski signs. In between spells motor power was restored. The blood pressure was $180 / 80$. Intravenous heparin was begun. On day 24 speech was slurred and the right limbs were slightly weak. On day 25 speech was severely slurred and the right limbs moved only slightly. On day 26 the right arm and leg were totally paralyzed and the left limbs were immobile save for very slight movement of the fingers and weak flexion and extension of the toes. The patient could not make a sound. She could not move her head or open her mouth. The eyelids closed feebly on command. The pupils were $2 \mathrm{~mm}$ in diameter and reacted to light. The eyes roved slowly from side to side, more fully to the left, without fixing and did not move to command. Between sweeps from side to side the eyeballs oscillated a few mm horizontally on either side of the central position and then paused briefly. Communication was carried out by having the patient signal with movements of the left toes and by this method she demonstrated that she was oriented to time and able to identify the examiner, do arithmetic and understand complicated instructions. The visual fields were full to finger movements. Spontaneous blinking was present. Sensation was intact to pinprick and joint position bilaterally. All tendon reflexes were brisk and the plantar responses were extensor bilaterally. The left limbs were spastic. The left arm could not be moved voluntarily and periodically without obvious stimulation underwent spasms of extensor posturing at the shoulder and elbow. The left leg did not participate in this decerebration. The jaw snapped shut when the lips were touched. On day 31 there was no movement anywhere except for the left toes which flexed and extended on command. She correctly identified the number of fingers held up before her. There was no change on day 32 . Only natural methods of treatment were used and the patient died on day 33 .

Both the left and right hemiplegias were of the pure motor type without sensory deficit, visual field defect, aphasia or apractognosia After the second stroke the patient was reduced to the "locked in" state. With the onset of the right hemiplegia the patient lost many of the movements of the left side that had returned in the three week interval after the onset of the left hemiplegia: abduction and adduction at the shoulder, flexion at the elbow, wrist, fingers and hip and extension at the knee. All voluntary movements of the face, eyes, tongue, neck and axial musculature ceased. Only slow flexion and extension movements of the left toes remained and these had been spared in the first stroke.

\section{Neuropathological Findings}

Gross examination of the brain disclosed no abnormalities of the surface. The arteries of the circle of Willis showed only slight atherosclerosis. Because it was planned to investigate the vascular lesions underlying the pure motor hemiplegias, the brain was not cut in the usual way but rather the cerebral hemispheres were sectioned horizontally beginning superiorly and proceeding inferiorly until the uppermost part of a capsular infarct was revealed on each side. The two blocks of basal ganglia with attached middle cerebral arteries were left undissected and sent for serial sectioning. The only other lesion in the rest of the brain was a $5 \times 5 \mathrm{~mm}$ lacunar infarct in the central white matter of the left upper parietal region, several $\mathrm{cm}$ removed from the motor system. The cerebral cortex was intact throughout as was the basis pontis.

Microscopic examination of the serial sections of the right basal ganglia disclosed a large $15 \times 15 \mathrm{~mm}$ recent infarct involving the posterior limb of the internal capsule, the adjacent body of the caudate nucleus and the lenticular nucleus (Figure 1). It measured approximately $24 \mathrm{~mm}$ vertically. The softening showed abundant macrophages consistent with an age of four weeks. Several vessels of the infarct were traced inferiorly where they converged to form a single artery of supply. In the lower $2 \mathrm{~mm}$ of the infarct this penetrating artery over a distance of $0.6 \mathrm{~mm}$ showed thinning and disorganization of the wall leaving only remnants of the internal elastic lamina. More proximally a normal appearing artery 500 microns in diameter took form although still occluded by fine connective tissue for about 2 $\mathrm{mm}$. Two $\mathrm{mm}$ still more proximally the artery joined the stem of the middle cerebral artery.

Microscopic examination of the serial sections of the left basal ganglia disclosed a large recent infarct of the posterior limb of the internal capsule, putamen and body of the caudate nucleus, measuring $15 \mathrm{~mm}$ anteroposteriorly, about $5 \mathrm{~mm}$ transversely and $25 \mathrm{~mm}$ vertically. The infarct showed early macrophage formation consistent with an age of 10 days. The small arteries supplying the infarcted territory were traced to 


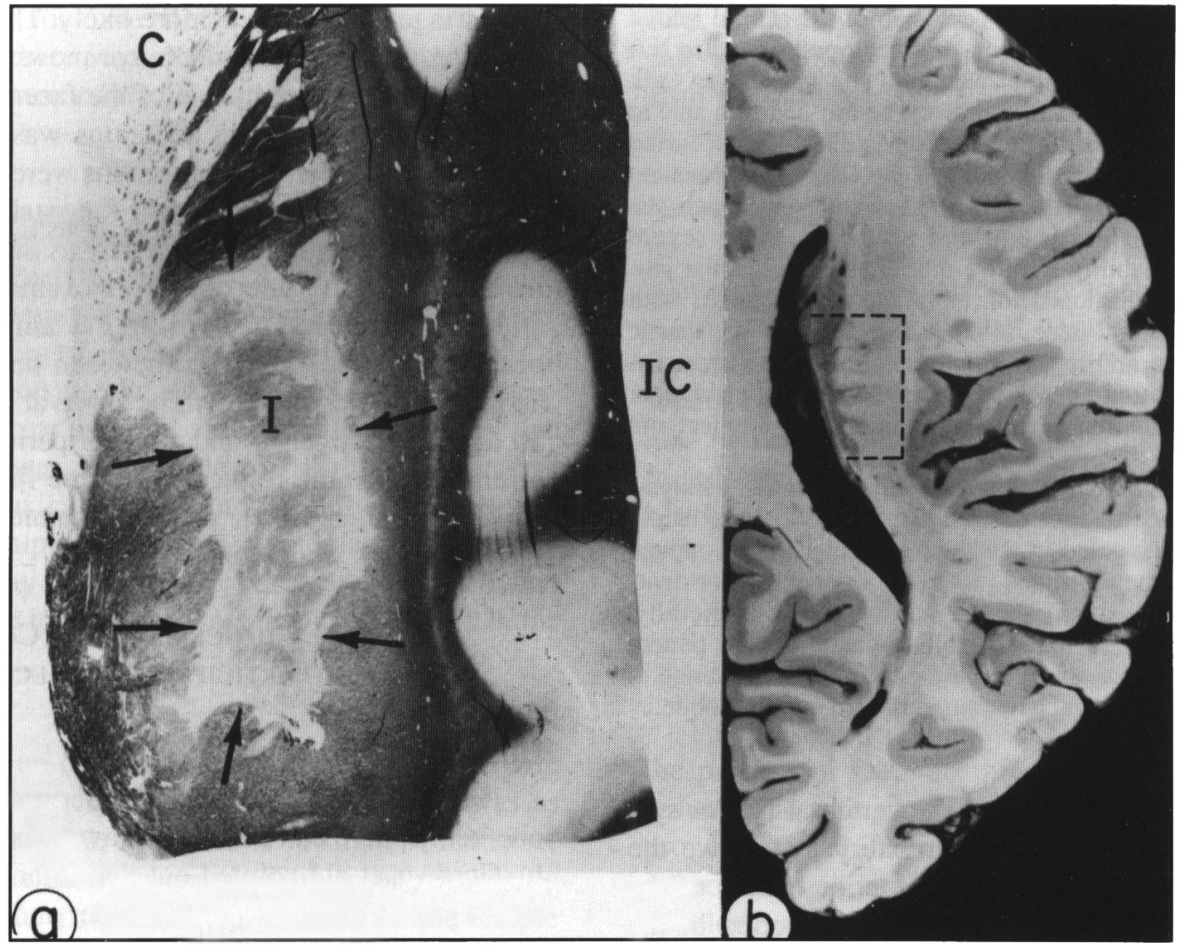

Figure 1-Patient 1. a) Myelin stain (Loyez) of horizontal section from the right upper internal capsule showing rectangular area of infarction. $(\times 2.2) . I=$ infarct $; C=$ head of caudate; $I C$ $=$ insular cortex. $b$ ) Horizontal section of a normal brain to illustrate the approximate site of the section in la.

their origin from the middle cerebral artery. About $2 \mathrm{~mm}$ distal to its origin from the middle cerebral artery, the penetrating artery measuring $\mathbf{5 0 0}$ microns in diameter was totally occluded by an atherosclerotic plaque that narrowed the lumen to about 50 microns and on which was superimposed a tiny pear-shaped fibrin microthrombus at the distal end of the stenosis. The plaque, which was 800 microns long, consisted of lipid-filled cells with a small admixture of fine fibrillar connective tissue.

\section{Comment on Patient 1}

The two major infarcts involved the posterior limb of the internal capsule on each side and were literally mirror-images (Figure 2). In their upper parts where the capsular fibers merged with the central white matter much of the posterior limb was affected while more inferiorly the posterior half of the posterior limb was involved. The cause of the infarct on the right side was an arterial lesion in which the vessel was severely disrupted by a process allied to lipohyalinosis while on the left side a minute fibrin thrombus was superimposed on a plaque of atherosclerosis that severely narrowed the artery. The capsular infarcts correlated well with the presence clinically of pure motor hemiplegias. $^{6}$ The vascular findings were similar to those in other cases of capsular hemiplegia. ${ }^{7}$

It seems clear that when the second or right hemiplegia developed on the 26th day the recovering function of the left side was also lost. Presumably the lesion in the left internal capsule caused not only the right hemiplegia but also re-paralysis of most of the left side. The existence of these two lesions widely separated in opposite hemispheres, apparently unrelated, discrete, deep, sparing the cortex and associated with a purely

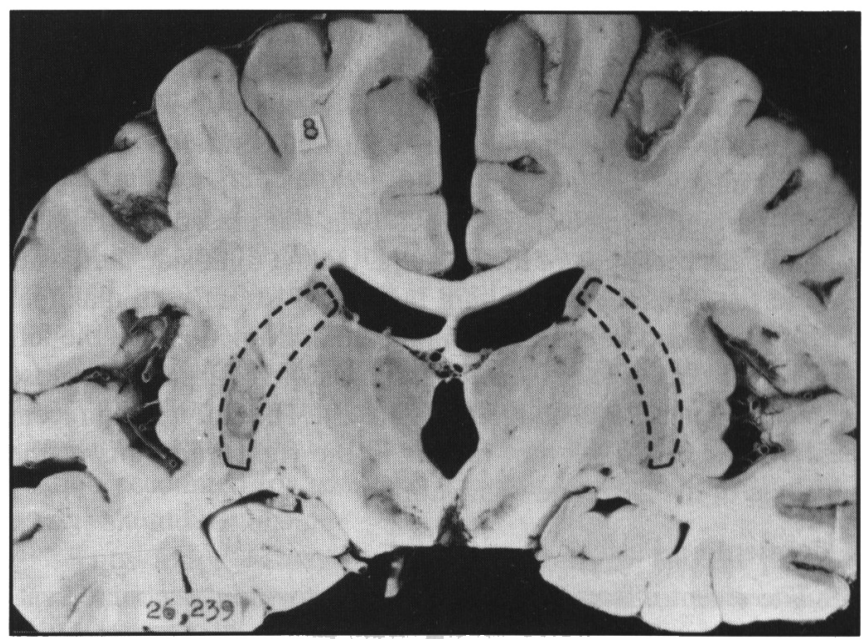

Figure 2 - Coronal cross section of a brain to illustrate with dotted outlines the approximate zone of infarction on each side.

motor paralysis in the presence of a lucid sensorium provides an unusual opportunity to examine the proposition that recovering function following a unilateral corticospinal lesion is subserved by the contralateral healthy corticospinal system. Based on the present data this is the only obvious conclusion.

Patient 2 A hypertensive woman aged 68, had a minor cerebrovascular episode six years before admission. Examination at that time showed slight dysarthria, incoordination of the left limbs, veering to the left, and hyperreflexia and an equivocal plantar response on the left side. Recovery was complete. Five years before admission she suddenly 
lost vision in the left eye as the result of occlusion of the central retinal artery. Seven months before admission she developed a severe left hemiparesis from which she slowly improved until she was able to walk using a portable metal walker and a foot brace. The left had was able to grasp the bars of the walker. Spasms of inappropriate laughter occurred at times. Two weeks before admission she had the first of 15 transient cerebral ischemic attacks each lasting 10 or 15 minutes in which she noted prickling of the right hand, arm and perioral region, severe dysarthria or mutism and inability to move the tongue or open the jaw. Examination on admission disclosed full extraocular movements with horizontal nystagmus on lateral gaze, blindness in the left eye, spastic hemiparesis of the left arm and leg, a slight reduction in the appreciation of pinprick and light touch on the left side and left Babinski sign. There was a slight incoordination of the right arm and leg. Intellect and memory were preserved.

Three days after admission the patient suddenly developed paralysis of all four limbs. She was alert and cooperative. Speech was a barely audible whisper, secretions pooled in the throat, dysphagia was almost absolute, the tongue could be protruded just to the lips, eye movements were full with horizontal nystagmus, the face was weak bilaterally, more on the left and there was a Babinski sign bilaterally. The patient survived 30 days during which time there was no important change in her neurological state. The four limbs remained flaccid and immobile.

\section{Neuropathological Findings}

There was severe atherosclerosis of the vertebral-basilar system with marked stenosis of the basilar artery just above the confluence of the vertebral arteries. The anterior branch of the left posterior inferior cerebellar artery was occluded in the region of the cerebellar tonsil. Coronal sections of the brain revealed several lesions which for the most part were old lacunar infarcts. There was one small old trabeculated lacune at each of the following sites: The right mid-thalamus, left medial thalamus, right mid-globus pallidus, the head of the caudate, right and left, left frontal white matter and the right and left upper basis pontis. There was old infarction of the medial surface of the right occipital lobe in the territory of the posterior cerebral artery. Both cerebral hemispheres showed scattered small old cortical infarcts.

Of particular relevance however was the presence of infarction in both pyramids of the medulla. On the right side there was an old fenestrated cavity $5 \mathrm{~mm}$ in vertical extent with maximal cavitation $9 \mathrm{~mm}$ below the pontomedullary junction. On the left side there was a recent disintegrating softening part of whose contents had been dislodged (Figure 3 ). The only other recent lesion was a $1 \times 1.5 \mathrm{~mm}$ infarct in the right mid-pons at the junction of the basis and tegmentum.

\section{Comment on Patient 2}

The recent infarct destroying the left pyramid accounted satisfactorily for the paralysis of the right arm and leg with relative sparing of the face and of ocular movement. The clinical observation that the limbs on both sides became permanently paralyzed at the same time suggested the likelihood that this infarct was also responsible for re-paralysis of the recovering left arm and leg, that is, the partial recovery of the old left hemiparesis was the result of the left or ipsilateral corticospinal system assuming some of the function of the first corticospinal system destroyed by the right pyramidal lesion. All but one of the other lesions found on pathological examination were old. The exception was a recent small infarct in the right mid-pons at the junction of the basis and the tegmentum too far posteriorly to have caused a hemiplegia. Moreover this lesion would have had to occur by chance at the same time as the left pyramidal lesion.
The first interpretation is more likely. The unusual pattern of the neurological deficit in which eye movements, jaw opening and movements of the right side of the face were spared at the same time as the buccolingual apparatus was severely involved also indicates that the pyramidal lesions were crucial.

These two cases seem ideal for establishing the principle of compensating contralateral activity as the basis of recovery from hemiplegia. The same observers examined the patients before and after the second hemiplegia and pathological studies became possible. The time between hemiplegias was 26 days and 7 months respectively. The survival times were brief -7 and 30 days respectively - and a longer period would have provided information on the re-recovery of the first hemiplegias. Maspes and $\mathrm{Pagni}^{2}$ staged their pedunculotomies 50-80 days apart and the follow-ups were "long term".

\section{Other Evidence Favoring Contralateral Participation in Recovery}

\section{Hemispherectomy}

Hemispherectomy provides incontrovertible evidence that one hemisphere can subserve movements of the limbs bilaterally. Gardner et al. ${ }^{8}$ pointed out that infantile hemiplegics usually retain good mobility at the shoulder and elbow along with some capacity to manipulate the thumb and flex and extend the fingers feebly. The spastic claw-hand of the elderly hemiplegic does not appear. Their gait is typical of spastic hemiplegia and often they retain some capacity to flex and extend the ankle joint and move the toes in plantar flexion. Hemispherectomy does not alter these functions and indeed improvement may occur. The remaining hemisphere and the brain stem must be responsible for the movements of the ipsilateral limbs. Bates ${ }^{9}$ electrically stimulated the mesial surface of the hemisphere remaining after hemispherectomy and elicited movement of the limbs on both sides. Gardner et al. presented the comparable data for 7 patients aged 9 to 45 years in whom hemispherectomy was performed for brain tumor. ${ }^{8}$ They regained sufficient strength in the leg to enable them to walk without aid. Voluntary

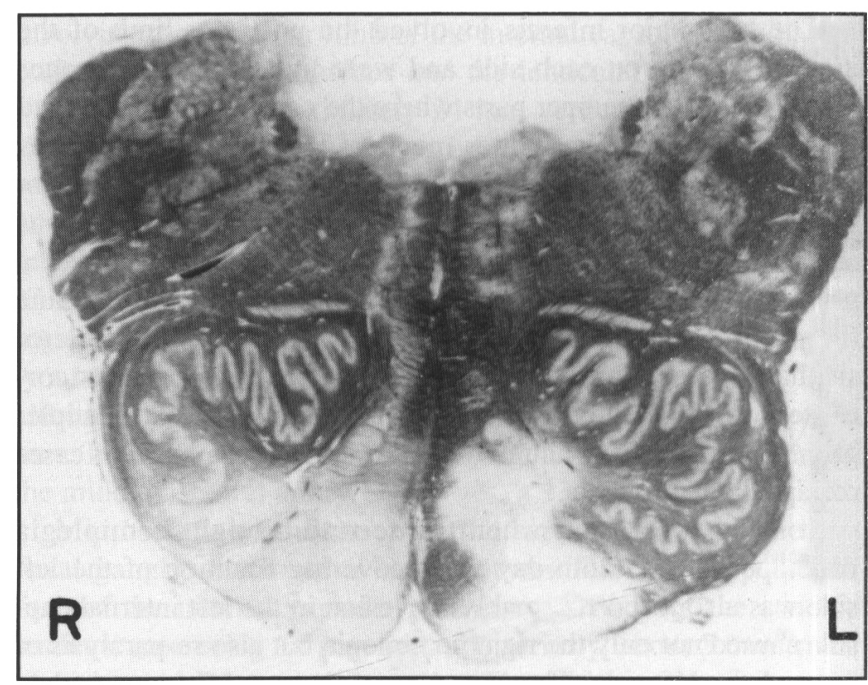

Figure 3-Patient 2. Myelin stain (Loyez) of section of the medulla oblongata showing bilateral pyramidal infarcts. $(\times 3)$ 
movements of ankle, toes, wrist, thumb and fingers never returned. Presumably there was movement at the shoulder and elbow. Any regained motor function appeared within hours or weeks.

\section{Hemiplegia}

Clinicopathologic correlations in cases of recovered hemiplegia also lend support. Foerster described a patient with hemiplegia resulting from thrombosis of the left middle cerebral artery who recovered the ability to use a pen in the affected hand.${ }^{10}$ On pathological examination myelin stains showed total degeneration of the left medullary pyramid and the author inferred that the homolateral tract was responsible for restitution of motor function. An analogous case was more recently reported. ${ }^{11} \mathrm{~A}$ woman with a left hemiplegia due to a pyramidal infarct recovered sufficiently to play the cello. On her death 19 years later autopsy showed almost total absence of myelinated fibers in the affected pyramid.

\section{Pedunculotomy}

Walker sectioned one cerebral peduncle in 9 patients with movement disorders. ${ }^{12}$ After initial paralysis, recovery was as follows: practically normal 2 , hemiparesis 7 , slight 4 , moderate 1 and severe 2 . One patient with hemiballismus, on awakening from the anesthetic had a right hemiplegia and spoke simple words. After 24 hours she moved the fingers feebly and the right leg fairly well. On day 5 she clenched the fingers feebly. On day 10 she walked without aid but dragged the leg. One year later she was doing her housework without difficulty.

Bucy et al. performed a right cerebral pedunculotomy in a man aged 70 who had a left hemiballismus of $2^{1 / 1} 2$ months duration. ${ }^{13}$ The incision in the central portion of the peduncle was 7 $\mathrm{mm}$ deep and $17 \mathrm{~mm}$ in length. Immediately after the operation there was a complete flaccid hemiplegia. In 24 hours slight voluntary movements were possible and on day 4 slight movements of the fingers and toes. On day 11 the patient could lift the arm above the head, move the leg with good power, stand alone and walk with assistance. On day 25 he walked with a "walker". On day 33 there was fairly good use of the hand, fine movements of the fingers and toes were possible and the patient walked unaided.There was a moderate left lower facial weakness. Recovery was maximal in 7 months at which time there was only a slight hemiparesis. The tendon reflexes were slightly hyperactive and there was little or no increase in tone. A Babinski sign was present. Sensation was intact. Hemiballismus did not return. The patient died $2^{1 / 2}$ years after the operation and pathological examination showed a lesion mainly of the medial one-half of the peduncle with sparing of some corticospinal fibers medially and posterior fibers laterally. It was estimated that descending degeneration involved 83 per cent of the corticospinal fibers of the pons and medulla. The degeneration extended into the spinal cord down to the conus medullaris. There was a 90 per cent loss of Betz cells in the precentral cortex. The question was whether the 17 per cent of fibers that were spared were adequate to account for the recovery.

\section{Subtle Bilaterality of Unilateral Motor Activity}

Electromyographic studies show that when one limb is in action its mate on the opposite side also participates. Cernacek found that on contracting the finger flexors and biceps on one side, electromyographic activity was recorded on the opposite side in $77.6 \%$ of healthy persons, $59.5 \%$ of hemiplegic limbs with no mobility and $87.7 \%$ of hemiplegic limbs with slight weakness. ${ }^{14} \mathrm{He}$ thought that irradiation from cortex to cortex via the corpus callosum was involved. Green reported crossed electromyographic activity in the biceps and triceps of normal children, young adults and hemiplegics. ${ }^{15} \mathrm{He}$ theorized that the anatomical basis was descending tracts from the brainstem.

In hemiplegics slight but definite motor impairment can be found in the arm on the "good" or normal side. Brodal in a personal observation noted that his left hemiplegia was accompanied by impairment of writing with his right hand. ${ }^{16}$ Jones et al. found motor impairment in the asymptomatic arm in 8 acute hemiplegics. ${ }^{17}$ Long ago Sherrington traced cortical motor fiber degeneration in one hemisphere to both sides of the spinal cord and to multiple segmental levels in Macaque. ${ }^{18}$

\section{Further Commentary}

The present thesis concerning contralateral participation in the process of recovery raises for speculation and discussion several interesting points. It would explain the relatively good prognosis in unilateral pure motor hemiplegia due to lacunar infarction in either the internal capsule or the brainstem and the hopeless prospect when the basis pontis is involved bilaterally in the "locked-in" syndrome. (The term pure motor hemiplegia is a clinical term and although involvement of the corticospinal tract is implied, the two are not to be equated or used interchangeably. Anatomically there may be silent extracorticospinal or extrapyramidal neural elements that play an inobvious role in contralateral participation in recovery. Discovery of these will require highly intricate studies).

The new information about recovery should be factored in, in clinical neurology to a greater extent than is usually the case. For example, some slowly growing tumors may permit motor compensation to occur, complicating topical diagnosis. The frequent devastating effect of lesions even of limited size in the second involved hemisphere is not fully appreciated. Seizures in recovered stroke patients may have idiosyncratic features. Mirror movements might well be reconsidered in the new light.

It might be inferred that recurrence of an isolated hemiplegia on the same side should not be possible if contralateral compensation accounted for recovery. Tandem lesions along the motor tracts should be tolerated.

Knowledge gained in the present study should provide insight into recovery in other systems: the amelioration of motor aphasia, ${ }^{19}$ the correction of ocular gaze preference, the recovery of memory function after infarction in the territory of the dominant posterior cerebral artery, the reversal of Wernicke's aphasia, the correction of cerebellar deficits, the subsidence of abulia after unilateral lesions, and so forth.

Now that a pathological basis has been demonstrated, the use of MRI should expedite further investigations of the recovery process. Virtually all stroke cases are potential contributors. The provision of a clearer conceptual framework should make the collection of precise neurological data more fruitful.

The stimulus or impetus for compensating activity in the contralateral motor system can only be surmised. How does the ipsilateral hemisphere 'sense' that one side of the face or one side of the body is no longer participating? One might postulate 
that a native built-in feed-back system is in continual life-long operation. If sensory afferents are involved, compensation would be hampered by the presence of a sensory loss, a point well-suited to methodical clinical investigation supported by MRI. In experimental hemisection of the spinal cord elimination of ipsilateral proprioception does not influence recovery. What constitutes the adequate stimulus is another facet. How severe must the deficit be and at what rate must it accrue in order that compensation be triggered?

Functional reorganization within the cerebral cortex is known to occur after peripheral nerve lesions ${ }^{20}$ and after amputation $^{21}$ and paraplegia. ${ }^{22}$ These findings are relevant when visualizing the possible neural basis of compensation after hemiplegia.

A few clinical features of the two cases presented should be mentioned. In both cases the herald of the second hemiplegia was transient ischemic attacks in which muteness was prominent. From experience in several other striking cases studied only clinically this appears to be a regular occurrence. In both cases the non-dominant side was involved first. Whether events follow the same course when the dominant side is first affected is not known. In the spinal cord cases of Nathan and Smith it made no difference which side was affected first. ${ }^{3}$

In Patient 1 the development of the second hemiplegia was accompanied by the appearance of spasticity and periodic extensor posturing at the shoulder and elbow on the recovering side. Maspes and Pagni drew particular attention to this reaction which they also noted on the first affected side when the second pedunculotomy was carried out. ${ }^{2}$ This phenomenon, not rare in clinical neurology, is witnessed particularly in progressing thrombosis of the basilar artery. It may well account for what appears to be seizure activity in the limbs of the first recovered hemiplegia at the time that a second hemiplegia occurs on the other side.

Sparing of movements of the left toes in both hemiplegias in Patient 1 means that compensation can be highly focal or regional. The pattern of movement when preserved normal activity and contralateral recovery activity are combined in adjacent parts should be of interest.

\section{Recovery of Motor Function After SPINAL CORD LESIONS}

Motor recovery after spinal cord lesions is inseparably linked to recovery after brain lesions. The remarkable recovery after stab or missile wounds involving one-half of the spinal cord was recognized many years ago by Rothmann who referred to three cases from the literature. ${ }^{23}$

Further evidence of good recovery in the spinal cord is provided by surgical experience. Putnam sectioned the pyramidal tract in the cervical spinal cord in the treatment of six cases of unilateral tremor. ${ }^{24}$ In general, the affected limbs which were initially paralyzed returned to approximately one-half their preoperative strength, movements of the individual fingers were possible and the right hand, if involved, could be used for writing. Walking was hemiparetic but satisfactory. Ebin sectioned not only the lateral corticospinal tract in the cervical region but also the opposite ventral corticospinal tract in 11 cases of paralysis agitans. ${ }^{25}$ After the combined lesions, paralysis lasted on the average 8 to 10 days, after which function gradually returned, appearing first in either the upper or lower extremity and either proximally or distally. In two cases return of motor function was no greater than $20 \%$, in eight it approximated 35 to $40 \%$ and in one case it was $90 \%$. Improvement occurred for three months. On the other hand, the associated sensory loss persisted. In neither of these reports were there pathological studies but the initial complete paralysis indicated involvement of the corticospinal system.

The extensive study of Nathan and Smith has already been referred to. ${ }^{3}$ Recovery of motor function after unilateral cordotomy was impressive. For example, in their case 93, the patient walked up and down stairs using one cane 38 days after operation. A second cordotomy contralaterally resulted in total paralysis of both legs, and at 40 days movement was still almost nil. The authors initially concluded that the corticospinal tract subserved recovery but a later analysis could not exclude a role for the reticulospinal tract (personal communication from Dr. Nathan).

The foregoing reports are pertinent in the interpretation of the clinical deficits associated with acute spinal cord disease. Slowly progressive disease seems also to elicit a compensation response, for in a review of the clinical findings in 80 cases of various types of spinal cord disease from personal files, none presented a unilateral paralysis at any stage of their illness, either by history or on examination. In patients with a progressive unilateral motor deficit, strength in the affected leg was always greater than $50 \%$ and adequate walking was possible until signs of involvement of the opposite side of the cord were evident. The two patients who most closely approached a Brown-Séquard syndrome had only a slight to moderate weakness of one leg combined with a crossed spinothalamic sensory loss. A classical Brown-Séquard syndrome must be a great rarity.

\section{The Results of Animal Experimentation}

The following brief, incomplete review will serve to illustrate that the results of surgical operations in animals may not readily apply in humans. For example, Travis and Woolsey reported that after ablation of the frontal motor fields bilaterally in Macaque, the animals can soon right themselves, sit, stand, walk and use the hands in feeding. ${ }^{26}$ After total decortication the animals can right themselves, get to their feet unaided, sit, stand and walk alone. These results are strikingly different from those encountered in the disease processes of humans.

Employing cortical ablation in Macaque, Murphy and Arana found no evidence for compensation by the contralateral hemisphere $^{27}$ whereas Ades and Raab demonstrated bilateral compensation from a unilateral ablation. ${ }^{28}$ Bucy et al., observing good recovery after staged bilateral pedunculotomy in rhesus monkeys, concluded that restitution depended on multineuronal pathways located outside of the cerebral peduncles. ${ }^{29}$ Walker and Richter from unilateral pedunculotomy in Macaque found no evidence of contralateral compensation. ${ }^{30}$ Tower's monkeys attempted standing and walking after lesions of the medullary pyramid bilaterally. ${ }^{31}$

From these results there emerges the impression that rules for motor behavior in humans must be developed from observations in the clinic.

Experimental lesions in the spinal cord of cat, dog and monkey evoke results comparable to those observed in human 
disease. Mettler and Liss concluded that recovery after unilateral spinal cord lesions in monkeys, was not due to hemispheric compensation. ${ }^{32}$ Here again nature's experiment in humans needs to be exploited.

\section{ACKNOWLEDGEMENT}

This study was supported by The Manny and Ruthy Cohen Foundation, Washington, D.C.

\section{REFERENCES}

1. Waxman SG. Functional recovery in diseases of the nervous system. In: Waxman SG, ed. Functional recovery in neurological disease. New York: Raven Press; Advances in Neurology, 1988; 47: $1-7$.

2. Maspes PE, Pagni CA. Surgical treatment of dystonia and choreoathetosis in infantile cerebral palsy by pedunculotomy: pathophysiological observations and therapeutic results. J Neurosurg 1964; 21 : 1076-1086.

3. Nathan PW, Smith MC. Effects of two unilateral cordotomies on the motility of the lower limbs. Brain 1973; 96: 471-494.

4. Chollet F, DiPiero V, Wise RJS, et al. The functional anatomy of motor recovery after stroke in humans: a study with positron emission tomography. Ann Neurol 1991; 29: 265-276.

5. Fisher CM. Bilateral capsular infarcts: the mechanism of recovery from hemiplegia. J Neuropathol Exp Neurol 1978; 37: 613.

6. Fisher CM, Curry HB. Pure motor hemiplegia of vascular origin. Arch Neurol 1965; 13: 30-44.

7. Fisher CM, Capsular infarcts: the underlying vascular lesions. Arch Neurol 1979; 36: 65-73.

8. Gardner WJ, Karnosh LJ, McClure CC, et al. Residual function following hemispherectomy for tumor and for infantile hemiplegia. Brain 1955; 78: 487-502.

9. Bastes JAV. Stimulation of the mesial surface of the human cerebral hemisphere after hemispherectomy. Brain 1953; 76: 405447.

10. Foerster O. 1. Restitution der motilität. Deutsche Ztschr Nervenh 1930; 115: 248-314

11. Ropper AH, Fisher CM, Kleinman GM. Pyramidal infarction in the medulla: a cause of pure motor hemiplegia sparing the face. Neurology 1979; 29: 91-95.

12. Walker AE. Cerebral pedunculotomy for involuntary movements. Surg Gynecol Obstet 1955; 100: 716-720.

13. Bucy PC, Keplinger JE, Siqueira EB. Destruction of the "pyramidal tract" in man. J Neurosurg $1964 ; 21: 385-398$.

14. Cernacek J. Contralateral motor irradiation-cerebral dominance: its changes in hemiparesis. Arch Neurol 1961; 4: 165-172.
15. Green JB. An electromyographic study of mirror movements Neurology 1967; 17: 91-94.

16. Brodal A. Self-observations and neuro-anatomical considerations after stroke. Brain 1973; 96: 675-694.

17. Jones RD, Donaldson IM, Parkin PJ. Impairment and recovery of ipsilateral sensory-motor function following unilateral cerebral infarction. Brain 1989; 112: 113-132.

18. Sherrington CS. On nerve-tracts degenerating secondarily to lesions of the cortex cerebri. J Physiol 1889; 10: 429-432.

19. Mohr JP. Rapid amelioration of motor aphasia. Arch Neurol 1973 28: 77-82.

20. Merzenich MM, Kaas JH, Nelson RJ, et al. Topographic reorganization of somatosensory cortical areas $3 \mathrm{~B}$ and 1 in adult monkeys following restricted deafferentation. Neuroscience 1983; 8: 33-55.

21. Merzenich MM, Nelson RJ, Stryker JP, et al. Somatosensory cortical map changes following digit amputation in adult monkeys. J Comp Neurol 1984; 224: 591-605.

22. Levy WJ, Amassian VE, Traad M, et al. Focal magnetic coil stimulation reveals motor cortical system reorganized in humans after traumatic quadriplegia. Brain Res 1990; 510: 130-134.

23. Rothmann M. Ueber die ergebnisse der Experimentellen ausschaltung der Motorischen Function und ihre Bedeutung fur die Pathologie. Ztschr Klin Med 1903; 48: 10-29.

24. Putnam TJ. Treatment of unilateral paralysis agitans by section of the lateral pyramidal tract. Arch Neurol Psychiatr 1940; 44: 950976.

25. Ebin J. Combined lateral and ventral pyramidotomy in treatment of paralysis agitans. Arch Neurol Psychiatr 1949; 62: 27-47.

26. Travis AM, Woolsey CN. Motor performance of monkeys after bilateral partial and total cerebral decortications. Am J Physiol Med 1956; 35: 273-310.

27. Murphy JP, Arana R. Extirpation of cortical arm area as defined by stimulation under conditions of primary facilitation (Macaca mulatta). J Neuropathol Exp Neurol 1947; 6: 194-200.

28. Ades HW, Raab DH. Recovery of motor function after two-stage extirpation of area 4 in monkeys (Macaca mulatta). J Physiol 1946; 9: 55-60.

29. Bucy PC, Ladpli R, Ehrlich A. Destruction of the pyramidal tract in the monkey. The effects of bilateral section of the cerebral peduncles. J Neurosurg 1966; 25: 1-23.

30. Walker AE, Richter H. Section of the cerebral peduncle in the monkey. Arch Neurol 1966; 14: 231-240.

31. Tower SS. Pyramidal lesion in the monkey. Brain 1940; 63: 36-90.

32. Mettler FA, Liss H. Functional recovery in primates after large subtotal spinal cord lesions. J Neuropathol Exp Neurol 1959; 18: 509-516. 